\title{
Antibiotic Resistance Pattern of the Blood Culture Isolates of Adult Sepsis Patients from a Rural Based Tertiary Care and Teaching Hospital, Piparia, Vadodara, India
}

\author{
Radhika Khara* and Sucheta J. Lakhani \\ Department of Microbiology, SBKS MI \& RC, Piparia, Waghodia, Gujarat, India \\ *Corresponding author
}

\begin{abstract}
A B S T R A C T
The rapid emergence of resistance against the antibiotics by bacteria poses a threat to the health and health benefits that can be obtained by the use of antibiotics. This problem is worldwide and it reflects on the overuse of these drugs and lack of development of new

Keywords

Sepsis, Blood

culture, Antibiotics,

Resistance

Article Info

Accepted:

22 April 2018

Available Online:

10 May 2018 drugs for combating this situation. Moreover infections due to such resistant bacteria add to the cost of healthcare in any nation. Thus performing antibiotic susceptibility and accordingly choosing the right antibiotic in each case to be treated invasive infections or non-invasive infections will prevent unjustified use of antibiotics as well as helps generate data regarding resistant bacteria and development of policies to prevent antibiotic resistance. A total of 673 blood cultures were performed from 743 clinically diagnosed sepsis patients. A total of 339 isolates were obtained from 330 (49.18\%) blood culture positive samples. Of the 339 isolates, 51.32\% (174/339) were Gram negative bacilli, $38.64 \%$ (131/339) were Gram positive bacteria and 10.02\% (34/339) Candida species. All the bacterial isolates, gram positive as well as gram negative were subjected to antibiotic susceptibility testing by Kirby-Bauer disc diffusion method. Least resistance was observed against cabapenems i.e. $21.8 \%$ with $10.26 \%$ against Imipenem and $11.54 \%$ against Ertapenem and Meropenem with Acinetobacter spp. showing the highest resistance i.e. $29.63 \%$ whereas the most was seen against Cefuroxime, Cefotaxime and Ceftazidime.
\end{abstract}

\section{Introduction}

The rapid emergence of resistance against the antibiotics by bacteria poses a threat to the health and health benefits that can be obtained by the use of antibiotics. This problem is worldwide and it reflects on the overuse of these drugs and lack of development of new drugs for combating this situation. Moreover infections due to such resistant bacteria add to the cost of healthcare in any nation (Ventola, 2015). Thus performing antibiotic susceptibility and accordingly choosing the right antibiotic in each case to be treated invasive infections or non-invasive infections will prevent unjustified use of antibiotics as well as helps generate data regarding resistant bacteria and development of policies to prevent antibiotic resistance.

This study was aimed at determining the antibiotic resistance pattern of the blood culture isolates of clinically diagnosed sepsis patients in our hospital. Also the same can be 
utilised for treatment of the patients already diagnosed and also to choose empirical treatment for the patients suspected having sepsis.

\section{Materials and Methods}

A total of 673 blood cultures were performed from 743 clinically diagnosed sepsis patients. Of these, 330 samples showed growth with 339 isolates - with $51.32 \%$ (174/339) Gram negative bacilli, 38.64\% (131/339) Gram positive bacteria and $10.02 \%$ (34/339) Candida species. Both the gram negative bacilli and gram positive cocci were subjected to antibiotic susceptibility testing by KirbyBauer Method (Collee et al., 14 ${ }^{\text {th }}$ edn, Betty A. Forbes et al., $12^{\text {th }}$ edn, Koneman et al., $6^{\text {th }}$ edn.) according to CLSI guidelines (CLSI, 2011).

AST was performed by modified Kirby-Bauer disk diffusion method. A well isolated colony or morphologically similar colonies were picked up with the help of sterile wire and mixed with sterile normal saline to prepare an inoculum of $0.5 \mathrm{McFarland}$. The mixture was then vortexed for homogenous turbidity.

A sterile swab was dipped into the prepared inoculum and it was ensured that extra solution was drained by rolling the swab against the wall of the tube. Using this swab, a lawn culture was prepared on Mueller Hinton Agar (HiMedia). By rotating the plate thrice at $60^{\circ}$ it was ensured that the whole surface is covered and uniform lawn culture is made. The antibiotic discs were placed on the plate within 15 minutes of inoculation and then the plates were incubated for 24 hours at $37^{\circ} \mathrm{C}$. The following antibiotic discs were used depending upon the isolate type:

\section{For Gram positive cocci}

Penicillin G (10 units), Erythromycin $(15 \mu \mathrm{g})$, Levofloxacin $(5 \mu \mathrm{g})$, Gentamicin $(10 \mu \mathrm{g})$,

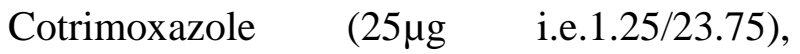
Vancomycin $(30 \mu \mathrm{g})$, Linezolid $(15 \mu \mathrm{g})$ and Doxycycline $(30 \mu \mathrm{g})$ were tested.

\section{For Gram negative bacilli}

Imipenem $(10 \mu \mathrm{g}), \quad$ Amikacin $(30 \mu \mathrm{g})$,

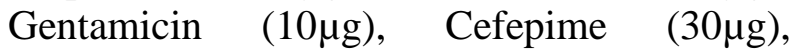
Cefuroxime $(30 \mu \mathrm{g})$, Ceftazidime $(30 \mu \mathrm{g})$, Cefotaxime $(30 \mu \mathrm{g})$ Ciprofloxacin $(5 \mu \mathrm{g})$, Amoxycillin + Clavulanic Acid $(30 \mu \mathrm{g}$ i.e $20 / 10 \mu \mathrm{g})$ and Cotrimoxazole $(25 \mu \mathrm{g}$ i.e. $1.25 / 23.75)$ ) were tested.

\section{For Pseudomonas spp.}

Imipenem $(10 \mu \mathrm{g}), \quad$ Piperacillin $(100 \mu \mathrm{g})$, Piperacillin + Tazobactam $(100 / 10 \mu g)$, Ceftazidime $(30 \mu \mathrm{g}), \quad$ Amikacin $(30 \mu \mathrm{g})$, Gentamicin $(10 \mu \mathrm{g})$ and Cefipime $(30 \mu \mathrm{g})$ were tested.

The observations were made by measuring the sizes of zones of inhibition around each disc using the zone scale (HiMedia) for each isolated organism. These zone sizes were then interpreted as Susceptible/Sensitive (S), Resistant (R) and Intermediate (I) according to the manufacturer's zone size interpretative chart which is as per the CLSI guidelines.

\section{VRSA detection: (CLSI, 2012 and 2013)}

As per CLSI, disc diffusion testing is not a reliable method for detection of vancomycin resistance. Hence MIC should be performed for all isolates which show no zone of inhibition around the disc before reporting it as resistant. Thus for detection of VRSA, MIC was performed using Vancomycin Ezy MIC strip (HiMedia) according to the manufacturer's instructions.

For this the colonies isolated and identified as Staphylococcus aureus were picked and inoculum of $0.5 \mathrm{McFarland}$ turbidity was prepared. A lawn culture was prepared using 
the above inoculum. With the help of the applicator stick provided with the package, the strip was placed on the centre of the plate and the plate was incubated for 18-24 hours. The control strains used: Enterococcus faecalis ATCC29212- Susceptible E. faecalis ATCC51299- Resistant as well as S. aureus ATCC 25923

The MIC was read where the ellipse intersected the MIC scale on the strip. These strips have a continuous gradient and thus MIC values may fall "in-between" two fold dilutions. In such cases the values were rounded up to the next two fold dilution before categorization as per the manufacturer's instructions.

According to CLSI the MIC values of $\leq 2 \mu \mathrm{g} / \mathrm{ml}$ as well as $\leq 4 \mu \mathrm{g} / \mathrm{ml}$ should be considered as susceptible while those with $\geq 8$ $\mu \mathrm{g} / \mathrm{ml}$ should be sent to the reference laboratory for confirmation before reporting as resistant.

\section{Results and Discussion}

The antibiotic resistance pattern of the major gram negative bacilli isolated from blood excluding Pseudomonas species, Proteus species, Citrobacter freundii and unidentified GNB is shown (in the Table 1 and Chart 1) below i.e. $(n=174-18=156)$. Least resistance was observed against cabapenems i.e. $21.8 \%$ with $10.26 \%$ against Imipenem and $11.54 \%$ against Ertapenem and Meropenem with Acinetobacter spp. showing the highest resistance i.e. $29.63 \%$ whereas the most was seen against Cefuroxime, Cefotaxime and Ceftazidime.

A total of $89.39 \%, 55.56 \%$ and $45.83 \%$ of Klebsiella spp., Acinetobacter spp. and E. coli showed resistance to amoxycilin-clavulanic acid. A total of $57.58 \%, 25.93 \%$ and $22.92 \%$ of Klebsiella spp., Acinetobacter spp. and E. coli showed resistance against Amikacin. Moreover, $93.94 \%, 96.30 \%$ and $87.50 \%$ of Klebsiella spp., Acinetobacter spp. and E. coli showed resistance to Cefuroxime and almost a similar percentage of resistance against Cefotaxime. The Salmonella species showed $100 \%$ susceptibility to Imipenem, Ertapenem/Meropenem, Gentamicin, Amikacin, Cefotaxime, Ceftazidime, Cefoxitin and Cefepime; but exhibited $20 \%$ resistance towards Cefurroxime and $6.66 \%$ resistance to Amoxycillin-Clavulanic acid and Ciprofloxacin.

Thus overall, least resistance was noted against imipenem (10.26\%) and ertapenem/ meropenem (11.54\%), slightly higher against cefepime $(44.87 \%)$, cotrimoxazole $(42.31 \%)$, amikacin $(35.90 \%)$ whereas highest was seen against cefuroxime $(85.26 \%)$ followed by cefotaxime $(79.49 \%)$, ceftazidime $(77.56 \%)$, ciprofloxacin $(74.36 \%)$ and gentamicin $(68.59 \%)$ as shown in the Chart 1

Pseudomonas spp. from blood showed much higher resistance to most of the antibiotics as compared to all other gram negative bacilli with as high as $50 \%$ resistance to Imipenem and Ertapenem/Meropenem both as shown in the Chart 2

A total of $38.64 \%(131 / 339)$ isolates were Gram positive bacteria including 2 Gram positive bacilli. Thus a total of $38.05 \%$ (129/339) were Gram positive cocci and of which $39.53 \%$ (51/129) were $S$. aureus, $37.98 \%$ (49/129) CoNS, $19.37 \%$ (25/129) Enterococcus species and $3.1 \%$ (4/129) were Streptococcus pyogenes. As shown (in the Chart 3) the maximum resistance was observed against Penicillin and Erythromicin whereas least was against Linezolid and Doxycycline. All the isolates showed 100\% susceptibility to Vancomycin. 
Table.1 Antibiotic resistance pattern of major Gram negative isolates of blood (\%)

\begin{tabular}{|c|c|c|c|c|c|c|c|c|c|c|c|c|}
\hline $\begin{array}{l}\text { Blood Cultures } \\
\text { Isolates }(\mathrm{n}=174 \text { - } \\
\qquad 18=156)\end{array}$ & IPM & AMC & AK & GEN & CPM & CIP & CXM & CTX & COT & CAZ & CX & $\begin{array}{l}\text { ERT/ } \\
\text { MRP }\end{array}$ \\
\hline $\begin{array}{l}\text { Klebsiella spp. } \\
\qquad(\mathrm{n}=66)\end{array}$ & 7.58 & 89.39 & 57.58 & 84.85 & 65.15 & 78.79 & 93.94 & 92.42 & 69.70 & 93.94 & 22.73 & 9.09 \\
\hline E. $\operatorname{coli}(n=48)$ & 6.25 & 45.83 & 22.92 & 64.58 & 31.25 & 83.33 & 87.50 & 85.42 & 20.83 & 79.17 & 37.50 & 6.25 \\
\hline $\begin{array}{c}\text { Acinetobacter } \\
\text { spp. }(\mathrm{n}=27)\end{array}$ & 29.63 & 55.56 & 25.93 & 74.07 & 44.44 & 85.19 & 96.30 & 81.48 & 37.04 & 77.78 & 40.74 & 33.33 \\
\hline $\begin{array}{c}\text { Salmonella } \\
\text { species }(\mathrm{n}=15)\end{array}$ & 0.00 & 6.67 & 0.00 & 0.00 & 0.00 & 6.67 & 20.00 & 0.00 & 0.00 & 0.00 & 0.00 & 0.00 \\
\hline
\end{tabular}

Chart.1 Total percentage of resistance exhibited by major Gram negative bacilli

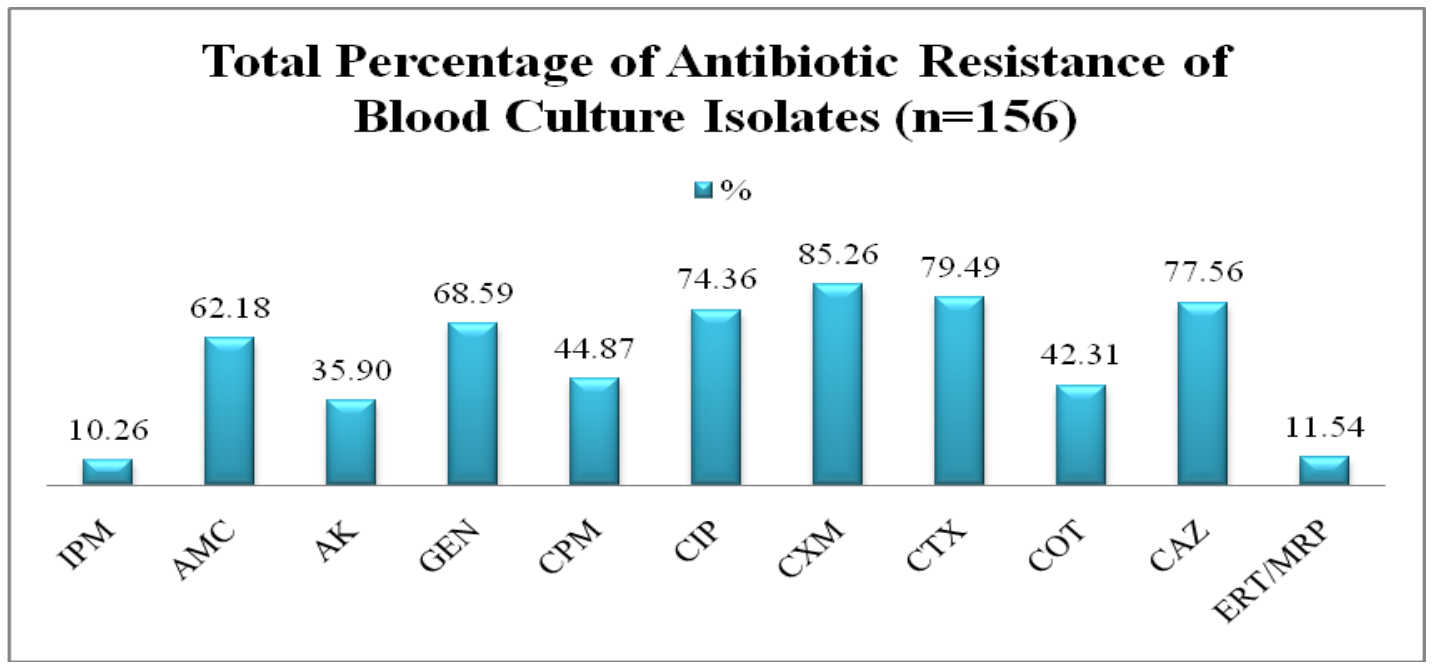

Chart.2 Antibiotic Resistance Pattern of Pseudomonas spp. $(\mathrm{n}=14)$

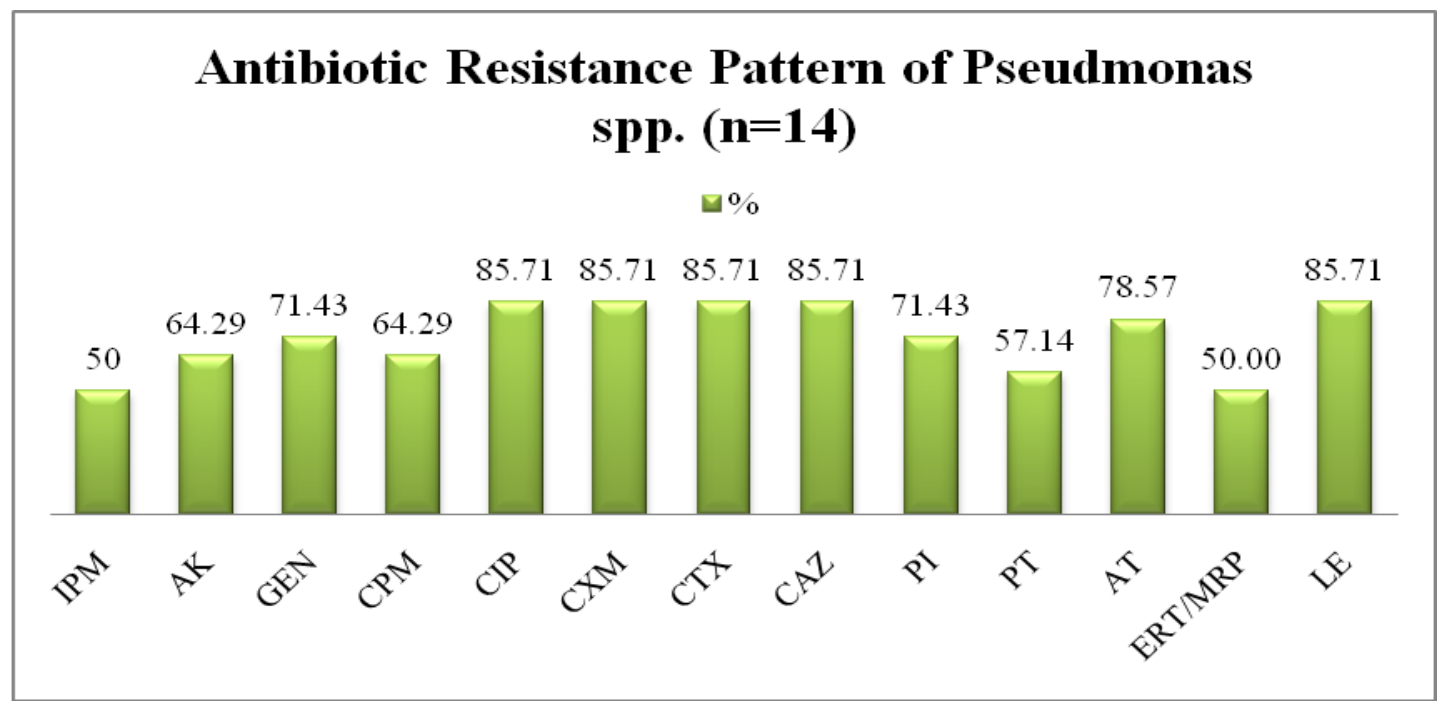


Chart.3 Antibiotic resistance pattern of Gram positive cocci from blood culture

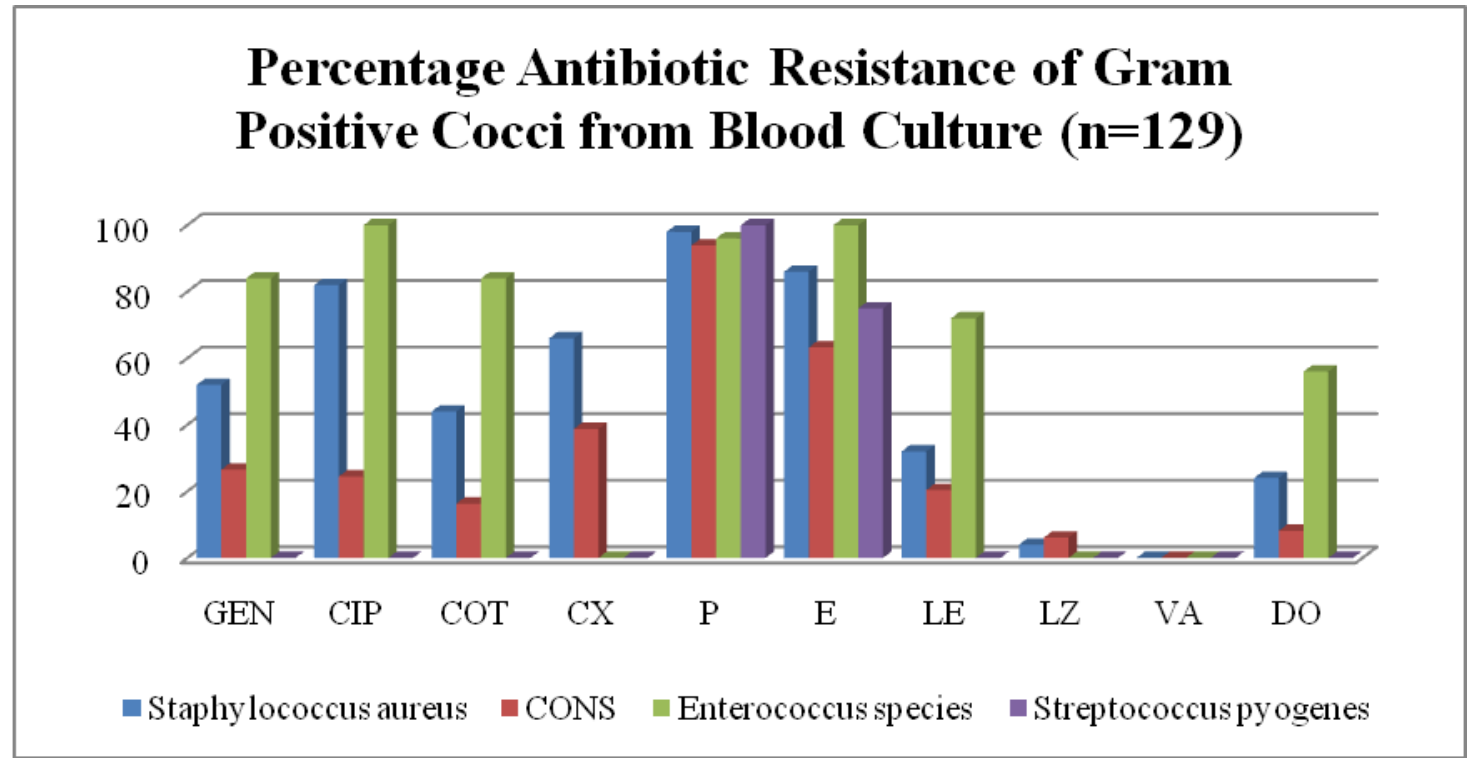

Chart.4 Total percentage of antibiotic resistance exhibited by Gram positive cocci $(\mathrm{n}=129)$

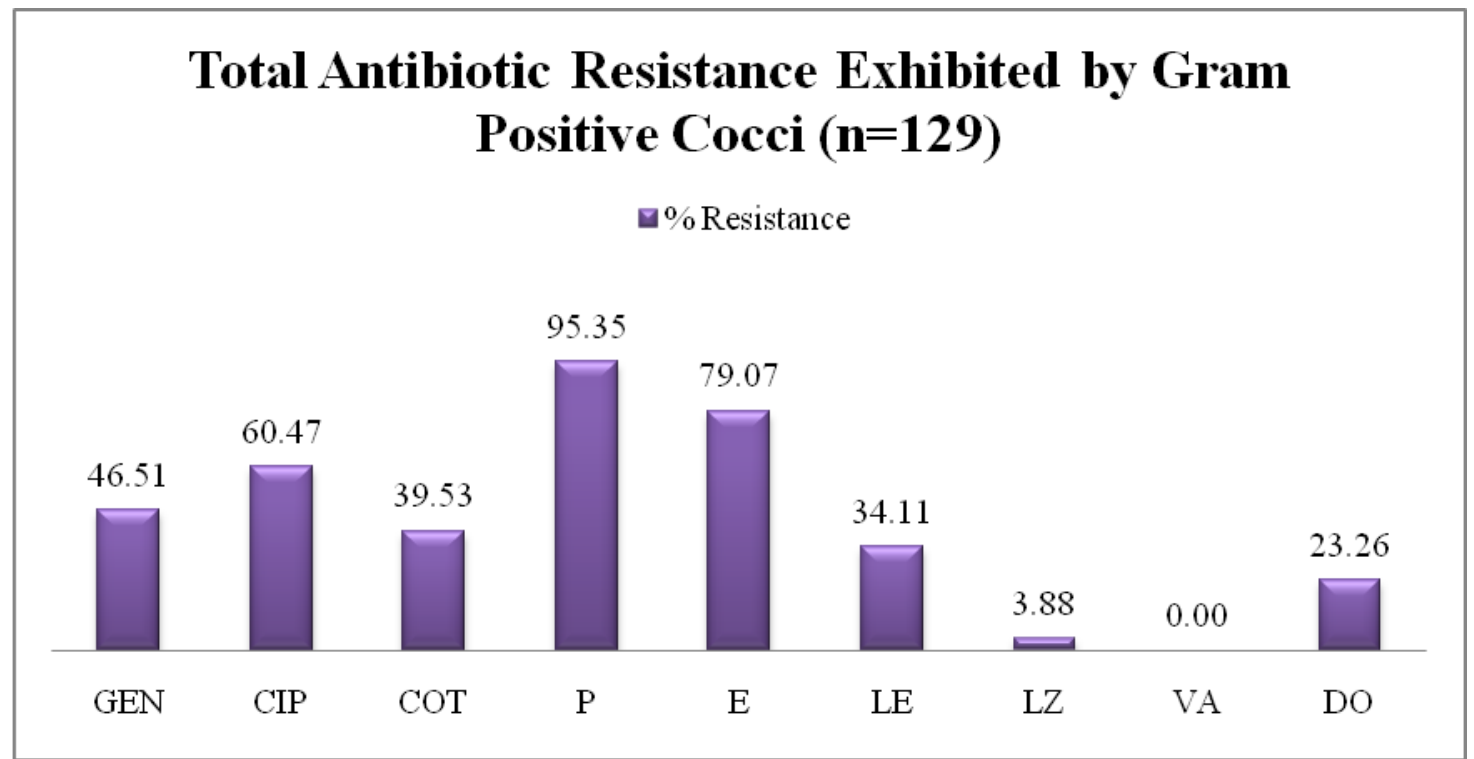

Moreover, the maximum resistance against most of the antibiotics was shown by Enterococcus species isolates.

Total of $52.94 \%$ (27/51) S. aureus were found to be MRSA and $34.69 \%$ (17/49) were found to be MRCONS. The antibiotic resistance pattern of the gram positive culture isolates of blood is as shown in the Chart 3 .
The overall resistance against antibiotics tested for gram positive cocci reveals $0 \%$ resistance towards vancomycin and $95.35 \%$ towards penicillin with varying resistance towards other antibiotics tested as shown in the Chart 4.

Thus overall least resistance was observed against carbapenems with Acinetobacter spp. 
showing the highest resistance i.e. $29.63 \%$. A total of $89.39 \%, 55.56 \%$ and $45.83 \%$ of Klebsiella spp., Acinetobacter spp. and E. coli showed resistance to amoxycilin/clavulanic acid. A total of $57.58 \%, 25.93 \%$ and $22.92 \%$ of Klebsiella spp., Acinetobacter spp. and E. coli showed resistance against Amikacin. Moreover, $93.94 \%, 96.30 \%$ and $87.50 \%$ of Klebsiella spp., Acinetobacter spp. and E. coli showed resistance to Cefuroxime and almost a similar percentage of resistance against Cefotaxime. Thus higher percentage of resistance against amoxicillin/clavulanic acid and $3^{\text {rd }}$ generation cephalosporins is comparable to the findings of Oza et al., (2016) from Surendranagar and Kante et al., (2014) from AP, India. Ugas et al., (2016) reports a case of septic shock with acute respiratory distress syndrome due to Salmonella typhi from Oklahoma, USA, which was found to be susceptible to ceftriaxone, cotrimoxazole and ciprofloxacin. Another case report from New Delhi, India by Randhawa VS et al., 2007, reports Salmonella paratyphiA, susceptible to Cefotaxime, Ciprofloxacin, Gentamicin, Amikacin, Cotrimoxazole which is similar to the findings of this study.

No resistance was observed against vancomycin and least towards linezolid (3.88\%) and doxycycline (23.26\%) whereas 97.42\% against Penicillin and $85.57 \%$ against Erythromycin with Enterococcus spp. showing the maximum resistance. These findings are again comparable to the findings of Oza et al., (2016) who report no resistance against vancomycin, linezolid and teicoplanin and also higher resistance by Enterococcus spp. towards penicillin. However, findings in regards to Penicillin and Erythromycin are in striking contrast to $100 \%$ susceptibility reported by Kante et al., (2014). Also resistance against ciprofloxacin was noted as $60.47 \%$ and $74.36 \%$ by GNB and GPC respectively. This is in contrast to the findings of Kumalo et al., (2016) who report ciprofloxacin to be $87.5 \%$ susceptible and as an effective antibiotic in their setup.

Thus it is important for any setup to perform antibiotic susceptibility testing of the isolates obtained which helps to generate data regarding resistance pattern of the isolates in any setup. We also saw an increased resistance against most of the antibiotics tested in our setup similar to the findings of the other studies. In our setup least resistance was seen against Imipenem amongst gram negative bacilli and against vancomycin amongst gram positive cocci rendering these as choice of treatment.

\section{References}

Betty, A., Forbes, Daniel, F., Sahm, Alice, S., Weissfeld. Bailey \& Scott's Diagnostic Microbiology. 12lh Ed, 216-247

Clinical Laboratory Standards Institute 2011. Performance Standards for antimicrobial susceptibility testing, $21^{\text {st }}$ informational supplement 2011. Document M100-S21, Vol.31, No. 1. Clinical Laboratory Standards Institute, Wayne, PA.

Clinical Laboratory Standards Institute 2012. Performance standards for antimicrobial susceptibility testing, $21^{\text {st }}$ informational supplement 2012. Document M07-A9. Clinical Laboratory Standards Institute, Wayne, PA.

Clinical Laboratory Standards Institute 2013. Performance standards for antimicrobial susceptibility testing, $21^{\text {st }}$ informational supplement 2013. Document M100S23. Clinical Laboratory Standards Institute, Wayne, PA.

Collee, J.G., Fraser, A.G., Marmion, B.P., Simmons, A. Mackie and McCartney Practical Medical Microbiology.14th Ed. 
Kante M, Uma P, John MS, Naidu MP. 2014. Bacterial profile of blood stream infections and antibiotic susceptibility pattern of isolates. Int $\mathrm{J}$ CurrMicrobiol App Sci, 3(12): 222-233.

Kumalo A, Kassa T, Mariam ZS, Daka D and Tadesse AH. 2016. Bacterial Profile of Adult Sepsis and their antimicrobial Susceptibility Pattern at Jimma University Specialized Hospital, South West Ethiopia. Health Science J; 10 (2):

Oza SS, Mehta SJ, Kikani KM, Oza SG. 2016. Bacteriological profile and antibiogram of blood culture isolates from patients of rural tertiary care hospital. Indian $\mathbf{J}$ Microbiology \& Mycology; 4 (3): 1-7.

Randhawa VS, Kumar A, Saili A, Datta V, Agrawal C and Mehta G. 2007.
Paratyphoid Sepsis. Indian J of Paediatr, 74 (2): 197-198.

Ugas MB, Carroll T, Kova L and Susana Chavez-Bueno. 2016. Salmonella Typhi-induced Septic Shock and Acute Respiratory Distress Syndrome in a previously healthy teenage patient treated with high dose dexamethasone. J of Investig Med High Impact Case Rep.; 4(2): 1-5.

Ventola CL. 2015. Antibiotic Resistance Crisis, Part 1: Causes \& Threat. P\&T; 40(4): 277-283.

Winn W, Allen S, Janda W, Koneman E, Procop G, Schreckenberger P, et al., 2006. Koneman's Colour Atlas \& Textbook of Diagnostic Microbiology, $6^{\text {th }}$ Edition.

\section{How to cite this article:}

Radhika Khara and Sucheta J. Lakhani. 2018. Antibiotic Resistance Pattern of the Blood Culture Isolates of Adult Sepsis Patients from a Rural Based Tertiary Care and Teaching Hospital, Piparia, Vadodara, India. Int.J.Curr.Microbiol.App.Sci. 7(05): 3363-3369. doi: https://doi.org/10.20546/ijcmas.2018.705.393 